\title{
Three families of two-parameter means constructed by trigonometric functions
}

\section{Zhen-Hang Yang*}

"Correspondence:yzhkm@163.com Power Supply Service Center, ZPEPC Electric Power Research Institute, Jianguozhong Road 219, Hangzhou, Zhejiang 310009, China

\begin{abstract}
In this paper, we establish three families of trigonometric functions with two parameters and prove their monotonicity and bivariate log-convexity. Based on them, three two-parameter families of means involving trigonometric functions, which include Schwab-Borchardt mean, the first and second Seiffert means, Sándor's mean and many other new means, are defined. Their properties are given and some new inequalities for these means are proved. Lastly, two families of two-parameter hyperbolic means, which similarly contain many new means, are also presented without proofs.
\end{abstract}

MSC: Primary 26E60; 26D05; secondary 33B10; $26 \mathrm{~A} 48$

Keywords: trigonometric function; hyperbolic function; mean; inequality

\section{Introduction}

Let $\mathbb{R}_{+}$denote the set of positive real numbers and $a, b \in \mathbb{R}_{+}$. A two-variable continuous function $M: \mathbb{R}_{+}^{2} \rightarrow \mathbb{R}_{+}$is called a mean on $\mathbb{R}_{+}$if

$$
\min (a, b) \leq M(a, b) \leq \max (a, b)
$$

holds. For convenience, however, we assume that $a \neq b$ in what follows unless otherwise stated.

There exist many elementary means. They can be divided into three classes according to main categories of basic elementary functions by their composition. The first class is mainly constructed by power functions, like the Stolarsky means [1] defined by

$$
S_{p, q}(a, b)= \begin{cases}\left(\frac{q\left(a^{p}-b^{p}\right)}{p\left(a^{q}-b^{q}\right)}\right)^{1 /(p-q)}, & p \neq q, p q \neq 0, \\ \left(\frac{a^{p}-b^{p}}{p(\ln a-\ln b)}\right)^{1 / p}, & p \neq 0, q=0, \\ \left(\frac{a^{q}-b^{q}}{q(\ln a-\ln b)}\right)^{1 / q}, & p=0, q \neq 0, \\ \exp \left(\frac{a^{p} \ln a-b^{p}}{a^{p}-b^{p}}-\frac{1}{p}\right), & p=q \neq 0, \\ \sqrt{a b}, & p=q=0\end{cases}
$$

and Gini means [2] defined by

$$
G_{p, q}(a, b)= \begin{cases}\left(\frac{a^{p}+b^{p}}{a^{q}+b^{q}}\right){ }^{1 /(p-q)}, & p \neq q ; \\ \exp \left(\frac{a^{p} \ln a+b^{1} \ln b}{a^{p}+b^{p}}\right), & p=q .\end{cases}
$$

\section{Springer}

○2013 Yang; licensee Springer. This is an Open Access article distributed under the terms of the Creative Commons Attribution License (http://creativecommons.org/licenses/by/2.0), which permits unrestricted use, distribution, and reproduction in any medium, provided the original work is properly cited. 
It is well known that the Stolarsky and Gini means are very important, they contain many famous means, for instance, $S_{1,0}(a, b)=L(a, b)$ - the logarithmic mean, $S_{1,1}(a, b)=I(a, b)$ - the identric (exponential) mean, $S_{2,1}(a, b)=G_{1,0}(a, b)=A(a, b), S_{2 p, p}(a, b)=G_{p, 0}(a, b)=$ $A^{1 / p}\left(a^{p}, b^{p}\right)=A_{p}$ - the $p$-order power mean, $S_{p, 0}(a, b)=L^{1 / p}\left(a^{p}, b^{p}\right)=L_{p}$ - the $p$-order logarithmic mean, $S_{p, p}(a, b)=I^{1 / p}\left(a^{p}, b^{p}\right)=I_{p}$ - the $p$-order identric (exponential) mean; $G_{2,0}(a, b)=Q(a, b)$ - the quadratic mean, $G_{1,1}(a, b)=Z(a, b)$ - the power-exponential mean, $G_{p, p}(a, b)=Z^{1 / p}\left(a^{p}, b^{p}\right)=Z_{p}$ - the $p$-order power-exponential mean, etc. The second class is mainly made up of exponential and logarithmic functions, such as the second part of Schwab-Borchardt mean (see [3], [4, Section 3, equation (2.3)], [5]) defined by

$$
S B=S B(a, b)= \begin{cases}\frac{\sqrt{b^{2}-a^{2}}}{\arccos (a b)}, & 0 \leq a<b, \\ \frac{\sqrt{a^{2}-b^{2}}}{\operatorname{arccosh}(a / b)}, & b<a,\end{cases}
$$

the logarithmic mean $L(a, b)$, the exponential mean defined by

$$
E(a, b)=\frac{b e^{b}-a e^{a}}{e^{b}-e^{a}}-1
$$

given in [6] (also see [7, 8]) by Sándor and Toader, and the Neuman-Sándor mean defined in [5] by

$$
N S=N S(a, b)=\frac{a-b}{2 \operatorname{arcsinh} \frac{a-b}{a+b}} .
$$

It should be noted that $N S$ is actually a Schwab-Borchardt mean since $N S(a, b)=S B(Q, A)$ mentioned by Neuman and Sándor in [5].

The third class is mainly composed of trigonometric functions and their inverses, for example, the first part of Schwab-Borchardt mean defined by (1.3), the first and second Seiffert means $[9,10]$ defined by

$$
\begin{aligned}
& P=P(a, b)=\frac{a-b}{2 \arcsin \frac{a-b}{a+b}}, \\
& T=T(a, b)=\frac{a-b}{2 \arctan \frac{a-b}{a+b}},
\end{aligned}
$$

respectively, and the new mean presented recently by Sándor in $[11,12]$ defined as

$$
X=X(a, b)=A e^{G / P-1},
$$

where $A=(a+b) / 2, G=\sqrt{a b}, P$ is defined by (1.5). As Neuman and Sándor pointed out in [5], the first and second Seiffert means are generated by the Schwab-Borchardt mean, because $S B(G, A)=P(a, b), S B(A, Q)=T(a, b)$.

From the published literature, the first and second classes have been focused on and investigated, and there are a lot of references (see [1,13-24]). While the third class is relatively little known.

The aim of this paper is to define three families of two-parameter means constructed by trigonometric functions, which include the Schwab-Borchardt mean $S B$, the first and second Seiffert means $P, T$, and Sándor's mean $X$. 
The paper is organized as follows. In Section 2, some useful lemmas are given. Three families of trigonometric functions and means with two parameters and their properties are presented in Sections 3-5. In Section 6, we establish some new inequalities for two-parameter trigonometric means. In the last section, two families of two-parameter hyperbolic means are also presented in the same way without proofs.

\section{Lemmas}

For later use, we give the following lemmas.

Lemma 2.1 [25, p.26] Let $f$ be a differentiable function defined on an interval I. Then the divided differences function $F$ defined on $I^{2}$ by

$$
F(x, y)=\frac{f(x)-f(y)}{x-y} \quad \text { if } x \neq y \quad \text { and } \quad F(x, x)=f^{\prime}(x)
$$

is increasing (decreasing) in both variables if and only iff is convex (concave).

Lemma 2.2 [26, Theorem 1] Let $f$ be a differentiable function defined on an interval I, and let $F$ be defined on $I^{2}$ by (2.1). Then the following statements are equivalent:

(i) $f^{\prime}$ is convex (concave) on $I$,

(ii) $F(x, y) \leq(\geq) \frac{f^{\prime}(x)+f^{\prime}(y)}{2}$ for all $x, y \in I$,

(iii) $F$ is bivariate convex (concave) on $I^{2}$.

Lemma 2.3 If $f:(-m, m) \rightarrow \mathbb{R}$ is a differentiable even function such that $f^{\prime}$ is convex in $(0, m)$, then the function $x \mapsto F(c+x, c-x)$ defined by (2.1) increases for positive $x$ if $c \geq 0$ and decreases if $c \leq 0$ provided $c+x, c-x \in(-m, m)$.

Proof Differentiation yields

$$
F^{\prime}(c+x, c-x)=\frac{\frac{f^{\prime}(c+x)+f^{\prime}(c-x)}{2}-\frac{f(c+x)-f(c-x)}{2 x}}{x}:=x^{-1} g_{c}(x) .
$$

Since $f$ is an even and differentiable function, it is easy to verify that $g_{c}(-x)=g_{c}(x), g_{-c}(x)=$ $-g_{c}(x)$. From this we only need to prove that for $c \geq 0, F^{\prime}(c+x, c-x)>0$ for $x \in(0, m)$ provided $c+x, c-x \in(-m, m)$ if $f^{\prime}$ is convex on $(0, m)$.

To this end, we first show two facts. Firstly, application of Lemma 2.2 leads to

$$
\begin{aligned}
\frac{f(c+x)-f(c-x)}{2 x} & =\frac{|c+x|-|c-x|}{2 x} \frac{f(|c+x|)-f(|c-x|)}{|c+x|-|c-x|} \\
& <\frac{2 c}{|c+x|+|c-x|} \frac{f^{\prime}(|c+x|)+f^{\prime}(|c-x|)}{2} .
\end{aligned}
$$

The second one states that if $h$ is a continuous and odd function on $[-d, d](d>0)$ and is convex on $[0, d]$, then, for $u, v \in(0, d]$ with $u>v$, the inequality

$$
\frac{h(u)}{u}>\frac{h(v)}{v}
$$

holds. Indeed, using the fact $h(0)=0$ and the property of a convex function, the second one easily follows. 
Now we can prove the desired result. When $x \in(0, m)$, application of the two previous facts and notice that $f^{\prime}$ is odd on $(-m, m)$ lead to

$$
\begin{aligned}
g_{c}(x) & >\frac{f^{\prime}(c+x)+f^{\prime}(c-x)}{2}-\frac{2 c}{|c+x|+|c-x|} \frac{f^{\prime}(|c+x|)+f^{\prime}(|c-x|)}{2} \\
& = \begin{cases}0 & \text { if } c-x \in[0, m), \\
-\frac{(c-x)(c+x)}{2 x}\left(\frac{f^{\prime}(c+x)}{x+c}-\frac{f^{\prime}(c-x)}{c-x}\right)>0 & \text { if } c-x \in(-m, 0) .\end{cases}
\end{aligned}
$$

Hence, $F^{\prime}(c+x, c-x)=x^{-1} g_{c}(x)>0$ for $c, x \geq 0$.

This completes the proof.

Lemma 2.4 The following inequalities are true:

$$
\begin{aligned}
& \sin x-x<(>) 0 \quad \text { for } x>(<) 0, \\
& \sin x-x \cos x>(<) 0 \quad \text { for } x \in(0, \pi) \quad(x \in(-\pi, 0)), \\
& x+\sin x>(<) 0 \quad \text { for } x>(<) 0, \\
& \cos x+x \sin x>1 \text { for } 0<|x|<\pi / 2, \\
& \cos x-\frac{\sin ^{3} x}{x^{3}}<0 \text { for } 0<|x|<\pi \\
& \frac{\sin x}{x}<\frac{2+\cos x}{3} \text { for } x \neq 0 .
\end{aligned}
$$

Proof Inequalities (2.2)-(2.5) easily follow by the elementary differential method, and we omit all details here. Inequality (2.6) can be derived from a well-known inequality given in [27, p.238]) by Adamović and Mitrinović for $0<|x|<\pi / 2$, while it is obviously true for $\pi / 2 \leq|x|<\pi$. Inequality (2.7) can be found in [28, Problem 5.11, 5.12]. This lemma is proved.

Lemma 2.5 [29, pp.227-229] Let $0<|x|<\pi$. Then we have

$$
\frac{1}{\sin x}=\frac{1}{x}+\sum_{n=1}^{\infty} \frac{2^{2 n}-2}{(2 n) !}\left|B_{2 n}\right| x^{2 n-1},
$$

where $B_{n}$ is the Bernoulli number.

\section{Two-parameter sine means}

\subsection{Two-parameter sine functions}

We begin with the form of hyperbolic functions of Stolarsky means defined by (1.1) to introduce the two-parameter sine functions. Let $t=\ln \sqrt{b / a}$. Then the Stolarsky means can be expressed in hyperbolic functions as

$$
S_{p, q}(a, b)=\sqrt{a b} \operatorname{Sh}(p, q, t)
$$


where

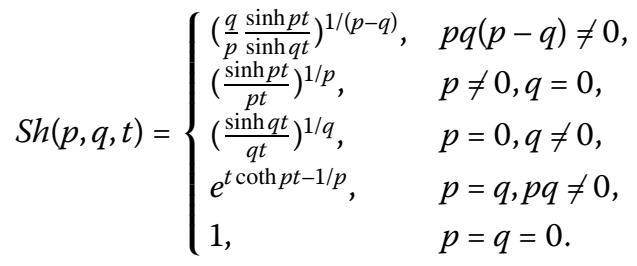

We call $\operatorname{Sh}(p, q, t)$ two-parameter hyperbolic sine functions. Accordingly, for suitable $p, q$, $t$, we can give the definition of sine versions of $\operatorname{Sh}(p, q, t)$ as follows.

Definition 3.1 The function $\tilde{S}$ is called a sine function with parameters if $\tilde{S}$ is defined on $[-2,2]^{2} \times(0, \pi / 2)$ by

$$
\tilde{S}(p, q, t)= \begin{cases}\left(\frac{q}{p} \frac{\sin p t}{\sin q t}\right)^{1 /(p-q)}, & p q(p-q) \neq 0, \\ \left(\frac{\sin p t}{p t}\right)^{1 / p}, & q=0, p \neq 0, \\ \left(\frac{\sin q t}{q t}\right)^{1 / q}, & p=0, q \neq 0, \\ e^{t \cot p t-1 / p}, & p=q \neq 0, \\ 1, & p=q=0 .\end{cases}
$$

$\tilde{S}$ is said to be a two-parameter sine function for short.

Now let us observe its properties.

Proposition 3.1 Let the two-parameter sine function $\tilde{S}$ be defined by (3.2). Then

(i) $\tilde{S}$ is decreasing in $p, q$ on $[-2,2]$, and is log-concave in $(p, q)$ for $(p, q) \in[0,2]^{2}$ and log-convex for $(p, q) \in[-2,0]^{2}$;

(ii) $\tilde{S}$ is decreasing and log-concave in $t$ on $(0, \pi / 2)$ for $p+q>0$, and is increasing and log-convex for $p+q<0$.

Proof We have

$$
\ln \tilde{S}(p, q, t)=\frac{f(p)-f(q)}{p-q} \quad \text { if } p \neq q \quad \text { and } \quad \ln \tilde{S}(p, p, t)=f^{\prime}(p)
$$

where

$$
f(x)=\ln \frac{\sin x t}{x} \quad \text { if }|x| \in(0,2] \text { and } \quad f(0)=\ln t .
$$

(i) We prove that $\tilde{S}$ is decreasing in $p, q$ on $[-2,2]$, and is log-concave in $(p, q)$ for $(p, q) \in$ $[0,2]^{2}$ and log-convex for $(p, q) \in[-2,0]^{2}$. By Lemmas 2.1 and 2.2, it suffices to check that $f$ is concave in $p, q \in[-2,2]$ and that $f^{\prime}$ is concave for $p, q \in[0,2]$ and convex for $p, q \in$ $[-2,0]$.

Differentiation and employing (2.2), (2.6) yield that for $t \in(0, \pi / 2)$,

$$
f^{\prime}(x)=\frac{t \cos t x}{\sin t x}-\frac{1}{x} \quad \text { if }|x| \in(0,2] \quad \text { and } \quad f^{\prime}(0)=0 \text {, }
$$




$$
\begin{aligned}
& f^{\prime \prime}(x)=\frac{\sin ^{2} t x-t^{2} x^{2}}{x^{2} \sin ^{2} t x}<0 \quad \text { for }|x| \in(0,2] \quad \text { and } \quad f^{\prime \prime}(0)=-\frac{t^{2}}{3}<0, \\
& f^{\prime \prime \prime}(x)=\frac{2 t^{3}}{\sin ^{3} t x}\left(\cos t x-\frac{\sin ^{3} t x}{t^{3} x^{3}}\right) \begin{cases}<0 & \text { if } x \in(0,2], \\
>0 & \text { if } x \in[-2,0),\end{cases}
\end{aligned}
$$

which prove part one.

(ii) Now we show that $\tilde{S}$ is decreasing and log-concave in $t$ on $(0, \pi / 2)$ for $p+q>0$, and increasing and log-convex for $p+q<0$. It is easy to verify that $f^{\prime}$ is an odd function on $[-2,2]$, and so $\ln \tilde{S}(p, q, t)$ can be written in the form of integral as

$$
\ln \tilde{S}(p, q, t)=\frac{1}{p-q} \int_{q}^{p} f^{\prime}(x) d x=\frac{p+q}{|p|+|q|} \frac{1}{|p|-|q|} \int_{|q|}^{|p|} f^{\prime}(x) d x
$$

Differentiation and application of (2.2) and (2.3) give

$$
\begin{aligned}
& \frac{\partial f^{\prime}}{\partial t}=\frac{1}{2 \sin ^{2} t x}(\sin 2 t x-2 t x)<0 \quad \text { for } x \in(0,2], \\
& \frac{\partial^{2} f^{\prime}}{\partial t^{2}}=\frac{2 x}{\sin ^{3} t x}(t x \cos t x-\sin t x)<0 \quad \text { for } x \in(0,2] .
\end{aligned}
$$

It follows from (3.7) that

$$
\begin{aligned}
& \frac{\partial \ln \tilde{S}}{\partial t}=\frac{p+q}{|p|+|q|} \frac{1}{|p|-|q|} \int_{|q|}^{|p|} \frac{\partial f^{\prime}}{\partial t} d x \begin{cases}<0 & \text { if } p, q \in[-2,2] \text { with } p+q>0, \\
>0 & \text { if } p, q \in[-2,2] \text { with } p+q<0,\end{cases} \\
& \frac{\partial^{2} \ln \tilde{S}}{\partial t^{2}}=\frac{p+q}{|p|+|q|} \frac{1}{|p|-|q|} \int_{|q|}^{|p|} \frac{\partial^{2} f^{\prime}}{\partial t^{2}} d x \begin{cases}<0 & \text { if } p, q \in[-2,2] \text { with } p+q>0, \\
>0 & \text { if } p, q \in[-2,2] \text { with } p+q<0,\end{cases}
\end{aligned}
$$

which proves part two and, consequently, the proof is completed.

From the proof of Proposition 3.1, we see that $f$ defined by (3.3) is an even function and $f^{\prime \prime \prime}(x)<0$ for $x \in[0,2]$ given by (3.6). Let $m=2$ and $c-x=p \in(-2,2)$. Then by Lemma 2.3 we immediately obtain the following.

Proposition 3.2 For fixed $c \in(-2,2)$, let $-\min (2,2-2 c) \leq p \leq \min (2,2 c+2)$ and $t \in$ $(0, \pi / 2)$, and let $\tilde{S}(p, q, t)$ be defined by (3.2). Then the function $p \mapsto \tilde{S}(p, 2 c-p, t)$ is decreasing on $[-2, c)$ and increasing on $(c, 2 c+2]$ for $c \in(-2,0]$, and is increasing on $[2 c-2, c)$ and decreasing on $(c, 2]$ for $c \in(0,2)$.

By Propositions 3.1 and 3.2 we can obtain some new inequalities for trigonometric functions.

Corollary 3.1 For $t \in(0, \pi / 2)$, we have

$$
\begin{aligned}
& \sqrt{\frac{\sin t}{t} \cos t}<\frac{2 \cos t+1}{3}<\exp (t \cot t-1)<\frac{1+\cos t}{2}<\frac{\sin t}{t}<\left(\frac{1+\cos t}{2}\right)^{1 / 3}, \\
& \cos ^{1 / 3} t<\left(\frac{2}{3} \cos t+\frac{1}{3}\right)^{1 / 2}<\left(\frac{1}{2} \cos t+\frac{1}{2} \cos \frac{t}{3}\right)^{3 / 5}
\end{aligned}
$$




$$
\begin{aligned}
& <\frac{\sin t}{t}<\left(\cos \frac{t}{5} \cos \frac{2 t}{5}\right)^{5 / 3}<\left(\frac{2}{3} \cos \frac{t}{2}+\frac{1}{3}\right)^{2} \\
& <\cos ^{3} \frac{t}{3}<\exp \left(t \frac{1+\cos t}{\sin t}-2\right)<\cos ^{4} \frac{t}{4}
\end{aligned}
$$

Proof (i) By Proposition 3.2, $\tilde{S}(p, 2-p, t)$ is increasing in $p$ on $[0,1)$ and decreasing on $[1,2]$, we have

$$
\tilde{S}(2,0, t)<\tilde{S}\left(\frac{3}{2}, \frac{1}{2}, t\right)<\tilde{S}(1,1, t) .
$$

Due to $\tilde{S}(1, q, t)$ is decreasing in $q$ on $[-2,2]$, we get

$$
\tilde{S}(1,1, t)<\tilde{S}\left(1, \frac{1}{2}, t\right)<\tilde{S}(1,0, t)<\tilde{S}\left(1,-\frac{1}{2}, t\right) .
$$

Simplifying leads to (3.8).

(ii) Similarly, since $p \mapsto \tilde{S}(p, 1-p, t)$ is increasing on $[-1,1 / 2)$ and decreasing on $(1 / 2,2]$, we get

$$
\begin{aligned}
\tilde{S}(2,-1, t) & <\tilde{S}\left(\frac{3}{2},-\frac{1}{2}, t\right)<\tilde{S}\left(\frac{4}{3},-\frac{1}{3}, t\right)<\tilde{S}(1,0, t) \\
& <\tilde{S}\left(\frac{4}{5}, \frac{1}{5}, t\right)<\tilde{S}\left(\frac{3}{4}, \frac{1}{4}, t\right)<\tilde{S}\left(\frac{2}{3}, \frac{1}{3}, t\right)<\tilde{S}\left(\frac{1}{2}, \frac{1}{2}, t\right),
\end{aligned}
$$

while $\tilde{S}\left(\frac{1}{2}, \frac{1}{2}, t\right)<\tilde{S}\left(\frac{1}{2}, \frac{1}{4}, t\right)$ follows by the monotonicity of $\tilde{S}(1 / 2, q, t)$ in $q$ on $[-2,2]$. Simplifying yields inequalities (3.9).

\subsection{Definition of two-parameter sine means and examples}

Being equipped with Propositions 3.1, 3.2, we can easily establish a family of means generated by (3.2). To this end, we have to prove the following statement.

Theorem 3.1 Let $p, q \in[-2,2]$, and let $\tilde{S}(p, q, t)$ be defined by (3.2). Then, for all $a, b>0$, $\mathcal{S}_{p, q}(a, b)$ defined by

$$
\begin{aligned}
& \mathcal{S}_{p, q}(a, b)=\max (a, b) \times \tilde{S}\left(p, q, \arccos \left(\frac{\min (a, b)}{\max (a, b)}\right)\right) \text { if } a \neq b \text { and } \\
& \mathcal{S}_{p, q}(a, a)=a
\end{aligned}
$$

is a mean of $a$ and $b$ if and only if $0 \leq p+q \leq 3$.

Proof Without lost of generality, we assume that $0<a \leq b$. Let $t=\arccos (a / b)$. Then the statement in question is equivalent to that the inequalities

$$
\cos t \leq \tilde{S}(p, q, t) \leq 1
$$

hold for $t \in(0, \pi / 2)$ if and only if $0 \leq p+q \leq 3$, where $\tilde{S}(p, q, t)$ is defined by (3.2). 
Necessity. We prove that the condition $0 \leq p+q \leq 3$ is necessary. If (3.11) holds, then we have

$$
\lim _{t \rightarrow 0^{+}} \frac{\ln \tilde{S}(p, q, t)-\ln \cos t}{t^{2}} \geq 0 \quad \text { and } \quad \lim _{t \rightarrow 0^{+}} \frac{\ln \tilde{S}(p, q, t)}{t^{2}} \leq 0 .
$$

Using power series extension gives

$$
\begin{aligned}
& \ln \tilde{S}(p, q, t)-\ln \cos t=-\frac{1}{6} t^{2}(p+q-3)+O\left(t^{4}\right), \\
& \ln \tilde{S}(p, q, t)=-\frac{1}{6} t^{2}(p+q)+O\left(t^{4}\right) .
\end{aligned}
$$

Hence we have

$$
-\frac{1}{6}(p+q-3) \geq 0 \quad \text { and } \quad-\frac{1}{6}(p+q) \leq 0,
$$

which implies that $0 \leq p+q \leq 3$.

Sufficiency. We show that the condition $0 \leq p+q \leq 3$ is sufficient. Clearly, $\max (p, q) \geq 0$. Now we distinguish two cases to prove (3.11).

Case 1: $p, q \geq 0$ and $p+q \leq 3$. This case can be divided into two subcases. In the first subcase of $(p, q) \in[0,2] \times[0,1]$ or $[0,1] \times[0,2]$, by the monotonicity of $\tilde{S}(p, q, t)$ in $p, q$ on $[-2,2]$, we get

$$
\cos t=\tilde{S}(2,1, t) \leq \tilde{S}(p, q, t) \leq \tilde{S}(0,0, t)=1
$$

In the second subcase of $(p, q) \in[1,2]^{2}$ and $p+q \leq 3$, it is derived that

$$
\tilde{S}(p, 3-p, t) \leq \tilde{S}(p, q, t) \leq \tilde{S}(0,0, t)=1 .
$$

From Proposition 3.2 it is seen that $\tilde{S}(p, 3-p, t)$ is increasing on $[1,3 / 2]$ and decreasing on $[3 / 2,2]$, which reveals that $\tilde{S}(p, 3-p, t)>\tilde{S}(2,1, t)=\cos t$, that is, the desired result.

Case 2: $p \geq 0, q \leq 0$ or $p \leq 0, q \geq 0$ and $p+q \leq 3$. Because of the symmetry of $p$ and $q$, we assume that $p \geq q$. Then $p \geq 0, q \leq 0$. Due to $p \in[0,2]$ and $p+q \leq 3$, we have $p \leq \min (3-q, 2)=2$. Using the monotonicity of $\tilde{S}(p, q, t)$ in $p, q$ on $[-2,2]$ again leads us to

$$
\tilde{S}(p, q, t) \geq \tilde{S}(2,0, t)=\sqrt{\frac{\sin 2 t}{2 t}}=\sqrt{\frac{\sin t}{t} \cos t}>\cos t .
$$

On the other hand, from $p+q \geq 0$, that is, $p \geq-q$, it is acquired that

$$
\tilde{S}(p, q, t) \leq \tilde{S}(-q, q, t)=1,
$$

which proves Case 2 and the sufficiency is complete.

Now we can give the definition of the two-parameter sine means as follows. 
Definition 3.2 Let $a, b>0$ and $p, q \in[-2,2]$ such that $0 \leq p+q \leq 3$, and let $\tilde{S}(p, q, t)$ be defined by (3.2). Then $\mathcal{S}_{p, q}(a, b)$ defined by (3.10) is called a two-parameter sine mean of $a$ and $b$.

As a family of means, the two-parameter sine means contain many known and new means.

Example 3.1 Clearly, for $0<a<b$, all the following

$$
\begin{aligned}
& \mathcal{S}_{1,0}(a, b)=\frac{\sqrt{b^{2}-a^{2}}}{\arccos (a / b)}=S B(a, b), \\
& \mathcal{S}_{1,1}(a, b)=b \exp \left(\frac{a}{S B(a, b)}-1\right), \\
& \mathcal{S}_{1 / 2,1 / 2}(a, b)=b \exp \left(\frac{a+b}{S B(a, b)}-2\right), \\
& \mathcal{S}_{3 / 2,1 / 2}(a, b)=b^{1 / 2}\left(\frac{2}{3} a+\frac{1}{3} b\right)^{1 / 2}
\end{aligned}
$$

are means of $a$ and $b$, where $S B(a, b)$ is the Schwab-Borchardt mean defined by (1.3).

To generate more means involving a two-parameter sine function, we need to note a simple fact: If $M_{1}, M_{2}, M$ are means of distinct positive numbers $x$ and $y$ with $M_{1}<M_{2}$, then $M\left(M_{1}, M_{2}\right)$ is also a mean and satisfies inequalities

$$
M_{1}<M\left(M_{1}, M_{2}\right)<M_{2}
$$

Applying the fact to Definition 3.2, we can obtain more means involving a two-parameter sine function, in which, as mentioned in Section 1, G, $A$ and $Q$ denote the geometric, arithmetic and quadratic means, respectively, and we have $G<A<Q$.

Example 3.2 Let $(a, b) \rightarrow(G, A)$. Then both the following

$$
\begin{aligned}
& \mathcal{S}_{1,0}(G, A)=S B(G, A)=\frac{a-b}{2 \arcsin \frac{a-b}{a+b}}=P(a, b), \\
& \mathcal{S}_{1,1}(G, A)=A \exp \left(\frac{G}{P}-1\right)=X(a, b)
\end{aligned}
$$

are means of $a$ and $b$, where $P=P(a, b)$ is the first Seiffert mean defined by $(1.5)$ and $X(a, b)$ is Sándor's mean defined by (1.7). Also, they lie between $G$ and $A$.

Example 3.3 Let $(a, b) \rightarrow(G, Q)$. Then both the following

$$
\begin{aligned}
& \mathcal{S}_{1,0}(G, Q)=S B(G, Q)=\frac{a-b}{\sqrt{2} \arctan \frac{a-b}{\sqrt{2 a b}}}:=U(a, b), \\
& \mathcal{S}_{1,1}(G, Q)=Q \exp \left(\frac{G}{U}-1\right)
\end{aligned}
$$

are means of $a$ and $b$, and between $G$ and $Q$. 
It is interesting that the new mean $U(a, b)$ is somewhat similar to the second Seiffert mean $T(a, b)$.

Example 3.4 Let $(a, b) \rightarrow(A, Q)$. Then both the following

$$
\begin{aligned}
& \mathcal{S}_{1,0}(A, Q)=S B(A, Q)=\frac{a-b}{2 \arctan \frac{a-b}{a+b}}=T(a, b), \\
& \mathcal{S}_{1,1}(A, Q)=Q \exp \left(\frac{A}{T}-1\right)
\end{aligned}
$$

are means of $a$ and $b$, where $T=T(a, b)$ is the second Seiffert mean defined by (1.6). Moreover, they are between $A$ and $Q$.

\subsection{Properties of two-parameter sine means}

From Propositions 3.1, 3.2 and Theorem 3.1, we easily obtain the properties of twoparameter sine means.

Property 3.1 The two-parameter sine means $\mathcal{S}_{p, q}(a, b)$ are symmetric with respect to parameters $p$ and $q$.

Property 3.2 The two-parameter sine means $\mathcal{S}_{p, q}(a, b)$ are decreasing in $p$ and $q$.

Property 3.3 The two-parameter sine means $\mathcal{S}_{p, q}(a, b)$ are log-concave in $(p, q)$ for $p, q>0$.

Property 3.4 The two-parameter sine means $\mathcal{S}_{p, q}(a, b)$ are homogeneous and symmetric with respect to $a$ and $b$.

Now we prove the monotonicity of two-parameter sine means in $a$ and $b$.

Property 3.5 Suppose that $0<a<b$. Then, for fixed $b>0$, the two-parameter sine means $\mathcal{S}_{p, q}(a, b)$ are increasing in $a$ on $(0, b)$. For fixed $a>0$, they are increasing in $b$ on $(a, \infty)$.

Proof (i) Let $t=\arccos (a / b)$. Then $\ln \mathcal{S}_{p, q}(a, b):=\ln b+\ln \tilde{S}(p, q, t)$. Differentiation yields

$$
\frac{\partial}{\partial a} \ln \mathcal{S}_{p, q}(a, b)=\frac{\partial}{\partial t} \ln \tilde{S}(p, q, t) \times \frac{\partial t}{\partial a}=-\frac{1}{\sqrt{b^{2}-a^{2}}} \frac{\partial}{\partial t} \ln \tilde{S}(p, q, t)
$$

which, by part two of Proposition 3.1, reveals that $\partial\left(\ln \mathcal{S}_{p, q}(a, b)\right) / \partial a>0$, that is, $\mathcal{S}_{p, q}(a, b)$ is increasing in $a$ on $(0, b)$.

(ii) Now we prove the monotonicity of $\mathcal{S}_{p, q}(a, b)$ in $b$. We have $\ln \mathcal{S}_{p, q}(a, b):=\ln a-$ $\ln \cos t+\ln \tilde{S}(p, q, t)$. Differentiation leads to

$$
\begin{aligned}
\frac{\partial}{\partial b} \ln \mathcal{S}_{p, q}(a, b) & =\left(\frac{\partial}{\partial t} \ln \tilde{S}(p, q, t)+\frac{\sin t}{\cos t}\right) \times \frac{\partial t}{\partial b} \\
& =\frac{a}{b \sqrt{b^{2}-a^{2}}}(H(p, q)+\tan t),
\end{aligned}
$$


where

$$
H(p, q):=\frac{h(p)-h(q)}{p-q} \text { if } p \neq q \text { and } H(p, p)=h^{\prime}(p),
$$

here

$$
h(x)=x \cot t x \quad \text { if }|x| \in(0,2] \quad \text { and } \quad h(0)=\frac{1}{t}
$$

is an even function on $[-2,2]$. Hence, to prove $\partial\left(\ln \mathcal{S}_{p, q}(a, b)\right) / \partial b>0$, it suffices to prove that for $p, q \in[-2,2]$ with $0 \leq p+q \leq 3$, the inequality $H(p, q)+\tan t>0$ is valid for $t \in$ $(0, \pi / 2)$. Differentiation again gives

$$
\begin{aligned}
& h^{\prime \prime}(x)=-2 t \frac{\sin t x-t x \cos t x}{\sin ^{3} t x}<0 \quad \text { for }|x| \in(0,2] \text { by }(2.3) \text { and } \quad h^{\prime \prime}(0)=-\frac{2 t}{3}<0, \\
& h^{\prime \prime \prime}(x)=-\frac{6 t^{3} x}{\sin ^{4} t x}\left(\frac{2+\cos 2 t x}{3}-\frac{\sin 2 t x}{2 t x}\right)<0 \quad \text { for } x \in(0,2] \text { by }(2.7) .
\end{aligned}
$$

It follows by Lemmas 2.1 and 2.3 that $H(p, q)$ is decreasing in $p$ and $q$ on $[-2,2]$ and $H(p, 3-$ $p)$ is increasing on $[1,3 / 2)$ and decreasing on $(3 / 2,2]$.

Next we distinguish two cases to prove $H(p, q)>0$ for $p, q \in[-2,2]$ with $0 \leq p+q \leq 3$.

Case 1: $p, q \geq 0$ and $p+q \leq 3$. This case can be divided into two subcases. In the first subcase of $(p, q) \in[0,2] \times[0,1]$ or $[0,1] \times[0,2]$, by the monotonicity of $H(p, q)$ in $p, q$ on $[-2,2]$, we have

$$
H(p, q)+\tan t \geq H(2,1)+\tan t=2 \cot 2 t-\cot t+\tan t=0 .
$$

In the second subcase of $(p, q) \in[1,2]^{2}$ and $p+q \leq 3$, it is derived from the monotonicities of $H(p, q)$ and $H(p, 3-p)$ that

$$
H(p, q)+\tan t \geq H(p, 3-p)+\tan t \geq H(2,1)+\tan t=0 .
$$

Case 2: $p \geq 0, q \leq 0$ or $p \leq 0, q \geq 0$ and $p+q \leq 3$. Because of the symmetry of $p$ and $q$, we assume that $p \geq q$. Then $p \geq 0, q \leq 0$. This together with $p, q \in[-2,2]$ with $p+q \leq 3$ gives $p \leq \min (3-q, 2)=2$. Therefore, we have

$$
H(p, q)+\tan t \geq H(2,0)+\tan t=\frac{1}{2}\left(2 \cot 2 t-\frac{1}{t}\right)+\tan t=\frac{2 t-\sin 2 t}{2 t \sin 2 t}>0,
$$

which proves the monotonicity of $\mathcal{S}_{p, q}(a, b)$ in $b$ on $(a, \infty)$ and the proof is complete.

Remark 3.1 Suppose that $0<a<b$. Then, by the monotonicity of $\mathcal{S}_{p, q}(a, b)$ in $a$ and $b$, we see that

$$
\mathcal{S}_{1,0}(G, A)<\mathcal{S}_{1,0}(G, Q)<\mathcal{S}_{1,0}(A, Q),
$$

which implies that

$$
P(a, b)<U(a, b)<T(a, b) .
$$


Similarly, we have

$$
X(a, b)=A \exp \left(\frac{G}{P}-1\right)<Q \exp \left(\frac{G}{U}-1\right)<Q \exp \left(\frac{A}{T}-1\right) .
$$

\section{Two-parameter cosine means}

\subsection{Two-parameter cosine functions}

In the same way, the Gini means defined by (1.2) can be expressed in hyperbolic functions by letting $t=\ln \sqrt{b / a}$ :

$$
G_{p, q}(a, b)=\sqrt{a b} C h(p, q, t),
$$

where

$$
C h(p, q, t)= \begin{cases}\left(\frac{\cosh p t}{\cosh q t}\right)^{1 /(p-q)}, & p \neq q, \\ e^{t \tanh p t}, & p=q \neq 0 \\ 1, & p=q=0 .\end{cases}
$$

We call $C h(p, q, t)$ two-parameter hyperbolic cosine functions. Analogously, we can define the two-parameter cosine functions as follows.

Definition 4.1 The function $\tilde{C}$ is called a two-parameter cosine function if $\tilde{C}$ is defined on $[-1,1]^{2} \times(0, \pi / 2)$ by

$$
\tilde{C}(p, q, t)= \begin{cases}\left(\frac{\cos p t}{\cos q t}\right)^{1 /(p-q)}, & p \neq q, \\ e^{-t \tan p t}, & p=q \neq 0, \\ 1, & p=q=0 .\end{cases}
$$

Similar to the proofs of Propositions 3.1 and 3.2, we give the following assertions without proofs.

Proposition 4.1 Let the two-parameter cosine function $\tilde{C}$ be defined by (4.2). Then

(i) $\tilde{C}$ is decreasing in $p, q$ on $[-1,1]$, and is log-concave in $(p, q)$ for $(p, q) \in[0,1]^{2}$ and $\log$-convex for $(p, q) \in[-1,0]^{2}$

(ii) $\tilde{C}$ is decreasing and log-concave in $t$ on $(0, \pi / 2)$ for $p+q>0$, and is increasing and log-convex for $p+q<0$.

Proposition 4.2 For fixed $c \in(-1,1)$, let $-\min (1,1-2 c) \leq p \leq \min (1,1+2 c)$ and $t \in$ $(0, \pi / 2)$, and let $\tilde{C}(p, q, t)$ be defined by (4.2). Then the function $p \mapsto \mathcal{C}(p, 2 c-p, t)$ is decreasing on $[-1, c)$ and increasing on $(c, 2 c+1]$ for $c \in(-1,0]$, and is increasing on $[2 c-1, c)$ and decreasing on $(c, 1]$ for $c \in(0,1)$.

Propositions 4.1 and 4.2 also contain some new inequalities for trigonometric functions, as shown in the following corollary.

Corollary 4.1 For $t \in(0, \pi / 2)$, we have

$$
\cos ^{2} \frac{t}{2}>\exp \left(-t \frac{1-\cos t}{\sin t}\right)>\left(2 \cos \frac{t}{2}-1\right)^{2}>\cos t
$$


Proof By Propositions 4.1 and 4.2 , we see that $\tilde{C}(1 / 2, q, t)$ is decreasing in $q$ on $[-1,1]$ and $\tilde{C}(p, 1-p, t)$ is decreasing in $p$ on $[0,1 / 2)$. It is obtained that

$$
\tilde{C}\left(\frac{1}{2}, 0, t\right)>\tilde{C}\left(\frac{1}{2}, \frac{1}{2}, t\right)>\tilde{C}\left(\frac{3}{4}, \frac{1}{4}, t\right)>\tilde{C}(1,0, t)
$$

which by some simplifications yields the desired inequalities.

\subsection{Definition of two-parameter cosine means and examples}

Similarly, by Propositions 4.1, 4.2, we can easily present a family of means generated by (4.2). Of course, we need to prove the following theorem.

Theorem 4.1 Let $p, q \in[-1,1]$, and let $\tilde{C}(p, q, t)$ be defined by (4.2). Then, for all $a, b>0$, $\mathcal{C}_{p, q}(a, b)$ defined by

$$
\begin{aligned}
& \mathcal{C}_{p, q}(a, b)=\max (a, b) \times \tilde{C}\left(p, q, \arccos \left(\frac{\min (a, b)}{\max (a, b)}\right)\right) \quad \text { if } a \neq b \text { and } \\
& \mathcal{C}_{p, q}(a, a)=a
\end{aligned}
$$

is a mean of $a$ and $b$ if and only if $0 \leq p+q \leq 1$.

Proof We assume that $0<a \leq b$ and let $t=\arccos (a / b)$. Then the desired assertion is equivalent to the inequalities

$$
\cos t \leq \tilde{C}(p, q, t) \leq 1
$$

hold for $t \in(0, \pi / 2)$ if and only if $0 \leq p+q \leq 1$, where $\tilde{C}(p, q, t)$ is defined by (4.2).

Necessity. If (4.5) holds, then we have

$$
\lim _{t \rightarrow 0^{+}} \frac{\ln \tilde{C}(p, q, t)-\ln \cos t}{t^{2}} \geq 0 \text { and } \lim _{t \rightarrow 0^{+}} \frac{\ln \tilde{C}(p, q, t)}{t^{2}} \leq 0 .
$$

Using power series extension gives

$$
\begin{aligned}
& \ln \tilde{C}(p, q, t)-\ln \cos t=-\frac{1}{2} t^{2}(p+q-1)+O\left(t^{4}\right), \\
& \ln \tilde{C}(p, q, t)=-\frac{1}{2} t^{2}(p+q)+O\left(t^{4}\right) .
\end{aligned}
$$

Hence we have

$$
-\frac{1}{2}(p+q-1) \geq 0 \quad \text { and } \quad-\frac{1}{2}(p+q) \leq 0,
$$

which yields $0 \leq p+q \leq 1$.

Sufficiency. We show that the condition $0 \leq p+q \leq 1$ is sufficient. Clearly, $\max (p, q) \geq 0$. Now we distinguish two cases to prove (4.5).

Case 1: $p, q \geq 0$ and $p+q \leq 1$. By Proposition 4.1 it is obtained that

$$
\tilde{C}(p, 1-p, t) \leq \tilde{C}(p, q, t) \leq \tilde{C}(0,0, t)=1 .
$$


From Proposition 4.2 it is seen that $\tilde{C}(p, 1-p, t)$ is increasing on $[0,1 / 2]$ and decreasing on $[1 / 2,1]$, which yields $\tilde{C}(p, 1-p, t)>\tilde{C}(1,0, t)=\cos t$, which proves Case 1 .

Case 2: $p \geq 0, q \leq 0$ or $p \leq 0, q \geq 0$ and $p+q \leq 1$. We assume that $p \geq q$. Then $p \geq 0$, $q \leq 0$. Due to $p \in[0,1]$ and $p+q \leq 1$, we have $p \leq \min (1-q, 1)=1$. Using the monotonicity of $\tilde{C}(p, q, t)$ in $p, q$ on $[-1,1]$ gives

$$
\tilde{C}(p, q, t) \geq \tilde{C}(1,0, t)=\cos t
$$

At the same time, since $p+q \geq 0$, that is, $p \geq-q$, we have

$$
\tilde{C}(p, q, t) \leq \tilde{C}(-q, q, t)=1
$$

which proves Case 2 and the sufficiency is complete.

Thus the two-parameter cosine means can be defined as follows.

Definition 4.2 Let $a, b>0$ and $p, q \in[-1,1]$ such that $0 \leq p+q \leq 1$, and let $\tilde{C}(p, q, t)$ be defined by (4.2). Then $\mathcal{C}_{p, q}(a, b)$ defined by (4.4) is called a two-parameter cosine mean of $a$ and $b$.

The two-parameter cosine means similarly include many new means, for example, when $0<a<b$,

$$
\mathcal{C}_{1 / 2,1 / 2}(a, b)=b \exp \left(\frac{a-b}{S B(a, b)}\right)
$$

is a mean, where $S B(a, b)$ is the Schwab-Borchardt mean defined by (1.3).

Additionally, let $(a, b) \rightarrow(G, A),(G, Q),(A, Q)$. Then all the following

$$
\begin{aligned}
& \mathcal{C}_{1 / 2,1 / 2}(G, A)=A \exp \left(\frac{G-A}{P}\right), \\
& \mathcal{C}_{1 / 2,1 / 2}(G, Q)=Q \exp \left(\frac{G-Q}{U}\right), \\
& \mathcal{C}_{1 / 2,1 / 2}(A, Q)=Q \exp \left(\frac{A-Q}{T}\right)
\end{aligned}
$$

are means of $a$ and $b$, where $P, T$ are the first and second Seiffert mean defined by (1.5) and (1.6), $U$ is defined by (3.16), and they lie between $G$ and $A, G$ and $Q, A$ and $Q$, respectively.

\subsection{Properties of two-parameter cosine means}

From Propositions 4.1, 4.2 and Theorem 4.1, we can deduce the properties of twoparameter cosine means as follows.

Property 4.1 $\mathcal{C}_{p, q}(a, b)$ are symmetric with respect to parameters $p$ and $q$.

Property 4.2 $\mathcal{C}_{p, q}(a, b)$ are decreasing in $p$ and $q$.

Property 4.3 $\mathcal{C}_{p, q}(a, b)$ are log-concave in $(p, q)$ for $p, q>0$. 
Property 4.4 $\mathcal{C}_{p, q}(a, b)$ are homogeneous and symmetric with respect to $a$ and $b$.

Property 4.5 Suppose that $0<a<b$. Then, for fixed $b>0$, the two-parameter cosine means $\mathcal{C}_{p, q}(a, b)$ are increasing in $a$ on $(0, b)$. For fixed $a>0$, they are increasing in $b$ on $(a, \infty)$

The proof of Property 4.5 is similar to that of Property 3.5, which is left to readers.

Remark 4.1 Assume that $0<a<b$. Then employing the monotonicity of $\mathcal{C}_{p, q}(a, b)$ in $a$ and $b$, we have

$$
G<A \exp \left(\frac{G-A}{P}\right)<Q \exp \left(\frac{G-Q}{U}\right)<Q \exp \left(\frac{A-Q}{T}\right)<Q .
$$

\section{Two-parameter tangent means}

\subsection{Two-parameter tangent functions}

Now we define the two-parameter tangent function and prove its properties, proofs of which are also the same as those of Propositions 3.1 and 3.2.

Definition 5.1 The function $\tilde{\mathcal{T}}$ is called a two-parameter tangent function if $\tilde{\mathcal{T}}$ is defined on $[-1,1]^{2} \times(0, \pi / 2)$ by

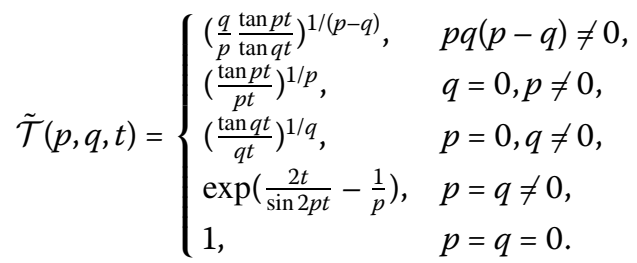

Proposition 5.1 Let the two-parameter tangent function $\tilde{\mathcal{T}}$ be defined by (5.1). Then

(i) $\tilde{\mathcal{T}}$ is increasing in $p, q$ on $[-1,1]$, and is log-convex in $(p, q)$ for $p, q>0$ and log-convex for $p, q<0$;

(ii) $\tilde{\mathcal{T}}$ is increasing and log-convex in $t$ for $p+q>0$, and is decreasing and log-concave for $p+q<0$.

Proof We have

$$
\ln \tilde{\mathcal{T}}(p, q, t)=\frac{g(p)-g(q)}{p-q} \quad \text { if } p \neq q \quad \text { and } \quad \ln \tilde{\mathcal{T}}(p, p, t)=g^{\prime}(p),
$$

where

$$
g(x)=\ln \frac{\tan t x}{x} \quad \text { if }|x| \in(0,1] \quad \text { and } \quad g(0)=\ln t .
$$

(i) To prove part one, by Lemmas 2.1 and 2.2 it suffices to check that $g$ is convex on $[-1,1]$ and $g^{\prime}$ is convex on $[0,1]$. In fact, differentiation and application of $(2.8)$ yield

$$
g^{\prime}(x)= \begin{cases}\frac{2 t}{\sin 2 t x}-\frac{1}{x} & \text { if }|x| \in(0,1],=\sum_{n=1}^{\infty} \frac{\left(2^{2 n}-2\right) 2^{2 n}}{(2 n) !}\left|B_{2 n}\right| t^{2 n} x^{2 n-1} . \\ 0 & \text { if } x=0\end{cases}
$$


Differentiation again gives

$$
\begin{aligned}
& g^{\prime \prime}(x)=\sum_{n=1}^{\infty} \frac{(2 n-1)\left(2^{2 n}-2\right) 2^{2 n}}{(2 n) !}\left|B_{2 n}\right| t^{2 n} x^{2 n-2}>0 \quad \text { for } x \in[-1,1], \\
& g^{\prime \prime \prime}(x)=\sum_{n=2}^{\infty} \frac{(2 n-1)(2 n-2)\left(2^{2 n}-2\right) 2^{2 n}}{(2 n) !}\left|B_{2 n}\right| t^{2 n} x^{2 n-3} \begin{cases}\geq 0 & \text { if } x \in[0,1], \\
\leq 0 & \text { if } x \in[-1,0] .\end{cases}
\end{aligned}
$$

Thus part one is proved.

(ii) For proving part two, we have to check that $\partial g^{\prime} / \partial t \geq 0$ and $\partial^{2} g^{\prime} / \partial t^{2} \geq 0$ for $x \in[0,1]$. Differentiating $g^{\prime}(x)$ given in (5.3) for $t$, we have

$$
\begin{aligned}
& \frac{\partial g^{\prime}}{\partial t}=\sum_{n=1}^{\infty} \frac{2 n\left(2^{2 n}-2\right) 2^{2 n}}{(2 n) !}\left|B_{2 n}\right| t^{2 n-1} x^{2 n-1} \geq 0 \quad \text { for } x \in[0,1] \\
& \frac{\partial^{2} g^{\prime}}{\partial t^{2}}=\sum_{n=1}^{\infty} \frac{2 n(2 n-1)\left(2^{2 n}-2\right) 2^{2 n}}{(2 n) !}\left|B_{2 n}\right| t^{2 n-2} x^{2 n-1} \geq 0 \quad \text { for } x \in[0,1]
\end{aligned}
$$

In the same method as the proof of part two in Proposition 3.1, part two in this proposition easily follows.

This completes the proof.

The following proposition is a consequence of Lemma 2.3, the proof of which is also the same as that of Proposition 3.2 and is left to readers.

Proposition 5.2 For fixed $c \in(-1,1)$, let $-\min (1,1-2 c) \leq p \leq \min (1,1+2 c)$ and $t \in$ $(0, \pi / 2)$, and let $\tilde{\mathcal{T}}(p, q, t)$ be defined by (5.1). Then the function $p \mapsto \tilde{\mathcal{T}}(p, 2 c-p, t)$ is increasing on $[-1, c)$ and decreasing on $(c, 1+2 c]$ for $c \in(-1,0]$, and is decreasing on $[2 c-1, c)$ and increasing on $(c, 1]$ for $c \in(0,1)$.

As an application of Propositions 5.1 and 5.2, we give the following corollary.

Corollary 5.1 For $t \in(0, \pi / 2)$, we have

$$
\left(\frac{\sin t}{t} \cos ^{-2} \frac{t}{2}\right)^{2}<\exp \left(\frac{2 t}{\sin t}-2\right)<\frac{\left(\frac{2}{3} \cos \frac{t}{2}+\frac{1}{3}\right)^{4}}{\left(\frac{2}{3} \cos t+\frac{1}{3}\right)^{2}}<\frac{\tan t}{t}
$$

Proof Propositions 5.1 and 5.2 indicate that $\tilde{\mathcal{T}}(1 / 2, q, t)$ is increasing in $q$ on $[-1,1]$ and $\tilde{\mathcal{T}}(p, 1-p, t)$ is decreasing in $p$ on $[0,1 / 2)$ and increasing on $(1 / 2,1]$. It follows that

$$
\tilde{\mathcal{T}}\left(\frac{1}{2}, 0, t\right)<\tilde{\mathcal{T}}\left(\frac{1}{2}, \frac{1}{2}, t\right)<\tilde{\mathcal{T}}\left(\frac{3}{4}, \frac{1}{4}, t\right)<\tilde{\mathcal{T}}(1,0, t),
$$

which, by some simplifications, yields the required inequalities.

\subsection{Definition of two-parameter tangent means and examples}

Before giving the definition of two-parameter tangent means, we firstly prove the following statement. 
Theorem 5.1 Let $p, q \in[-1,1]$, and let $\tilde{\mathcal{T}}(p, q, t)$ be defined by (5.1). Then, for all $a, b>0$, $\mathcal{T}_{p, q}(a, b)$ defined by

$$
\begin{aligned}
& \mathcal{T}_{p, q}(a, b)=\min (a, b) \times \tilde{\mathcal{T}}\left(p, q, \arccos \left(\frac{\min (a, b)}{\max (a, b)}\right)\right) \text { if } a \neq b \text { and } \\
& \mathcal{T}_{p, q}(a, a)=a
\end{aligned}
$$

is a mean of $a$ and $b$ if $0 \leq p+q \leq 1$.

Proof We assume that $0<a \leq b$ and let $t=\arccos (a / b)$. Then $\mathcal{T}_{p, q}(a, b)$ is a mean of $a$ and $b$ if and only if the inequalities

$$
1 \leq \tilde{\mathcal{T}}(p, q, t) \leq \frac{1}{\cos t}
$$

hold for $t \in(0, \pi / 2)$, where $\tilde{\mathcal{T}}(p, q, t)$ is defined by (5.1). Similarly, it can be divided into two cases.

Case 1: $p, q \geq 0$ and $p+q \leq 1$. From the monotonicity of $\mathcal{T}_{p, q}(a, b)$ in $p, q$ on $[-1,1]$, it is deduced that

$$
1=\tilde{\mathcal{T}}(0,0, t) \leq \tilde{\mathcal{T}}(p, q, t) \leq \tilde{\mathcal{T}}(p, 1-p, t) .
$$

By Proposition 5.2 we can see that $\tilde{\mathcal{T}}(p, 1-p, t)$ is decreasing on $[0,1 / 2]$ and increasing on $[1 / 2,1]$, which yields

$$
\tilde{\mathcal{T}}(p, 1-p, t)<\tilde{\mathcal{T}}(1,0, t)=\frac{\tan t}{t}=\frac{\sin t}{t} \frac{1}{\cos t}<\frac{1}{\cos t},
$$

that is, the desired result.

Case 2: $p \geq 0, q \leq 0$ or $p \leq 0, q \geq 0$ and $p+q \leq 1$. We assume that $p \geq q$. Analogously, there must be $p \leq \min (1-q, 1)=1$. Using the monotonicity of $\tilde{\mathcal{T}}(p, q, t)$ in $p, q$ on $[-1,1]$ gives

$$
\tilde{\mathcal{T}}(p, q, t) \leq \tilde{\mathcal{T}}(1,0, t)=\frac{\tan t}{t}<\frac{1}{\cos t} .
$$

Noticing that $p+q \geq 0$, that is, $p \geq-q$, we have

$$
\tilde{\mathcal{T}}(p, q, t) \geq \tilde{\mathcal{T}}(-q, q, t)=1
$$

which proves Case 2 and the proof is finished.

We are now in a position to define the two-parameter tangent means by (5.1).

Definition 5.2 Let $a, b>0$ and $p, q \in[-1,1]$ such that $0 \leq p+q \leq 1$, and let $\tilde{\mathcal{T}}(p, q, t)$ be defined by (5.1). Then $\mathcal{T}_{p, q}(a, b)$ defined by (5.5) is called a two-parameter tangent mean of $a$ and $b$.

Here are some examples of two-parameter tangent means. 
Example 5.1 For $0<a<b$, both the following

$$
\begin{aligned}
& \mathcal{T}_{1,0}(a, b)=\frac{\sqrt{b^{2}-a^{2}}}{\arccos (a / b)}=S B(a, b), \\
& \mathcal{T}_{1 / 2,1 / 2}(a, b)=a \exp \left(\frac{2 b}{S B(a, b)}-2\right)
\end{aligned}
$$

are means of $a$ and $b$, where $S B(a, b)$ is the Schwab-Borchardt mean defined by (1.3).

Example 5.2 Let $(a, b) \rightarrow(G, A),(G, Q),(A, Q)$. Then all the following

$$
\begin{aligned}
& \mathcal{T}_{1 / 2,1 / 2}(G, A)=G \exp \left(\frac{2 A}{P}-2\right), \\
& \mathcal{T}_{1 / 2,1 / 2}(G, Q)=G \exp \left(\frac{2 Q}{U}-2\right), \\
& \mathcal{T}_{1 / 2,1 / 2}(A, Q)=A \exp \left(\frac{2 Q}{T}-2\right)
\end{aligned}
$$

are means of $a$ and $b$, where $P, T$ are the first and second Seiffert mean defined by (1.5) and (1.6), $U$ is defined by (3.16). Also, they lie between $G$ and $A, G$ and $Q, A$ and $Q$, respectively.

\subsection{Properties of two-parameter tangent means}

From Propositions 5.1 and 5.2 and Theorem 5.1, we see that the properties of twoparameter tangent means are similar to those of sine ones.

Property 5.1 $\mathcal{T}_{p, q}(a, b)$ is symmetric with respect to parameters $p$ and $q$.

Property 5.2 $\mathcal{T}_{p, q}(a, b)$ is increasing in $p$ and $q$.

Property 5.3 $\mathcal{T}_{p, q}(a, b)$ is log-convex in $(p, q)$ for $p, q>0$.

Property 5.4 $\mathcal{T}_{p, q}(a, b)$ is homogeneous and symmetric with respect to $a$ and $b$.

Now we prove the monotonicity of two-parameter trigonometric means in $a$ and $b$.

Property 5.5 Let $0<a<b$. Then, for fixed $a>0$, the two-parameter tangent mean $\mathcal{T}_{p, q}(a, b)$ is increasing in $b$ on $(a, \infty)$. For fixed $b>0$, the two-parameter tangent mean $\mathcal{T}_{p, q}(a, b)$ is increasing in $a$ on $(0, b)$.

Proof (i) Let $t=\arccos (a / b)$. Then $\ln \mathcal{T}_{p, q}(a, b):=\ln a+\ln \tilde{\mathcal{T}}(p, q, t)$. Differentiation yields

$$
\frac{\partial}{\partial b} \ln \mathcal{T}_{p, q}(a, b)=\frac{\partial}{\partial t} \ln \tilde{\mathcal{T}}(p, q, t) \times \frac{\partial t}{\partial b}=\frac{a}{\sqrt{b^{2}-a^{2}}} \frac{\partial}{\partial t} \ln \tilde{\mathcal{T}}(p, q, t)
$$

Application of Proposition 5.1 yields $\partial\left(\ln \mathcal{T}_{p, q}(a, b)\right) / \partial b>0$, which proves part one. 
(ii) Now we prove the monotonicity of $\mathcal{T}_{p, q}(a, b)$ in $a$. Since $\ln \mathcal{T}_{p, q}(a, b)$ can be written as $\ln \mathcal{T}_{p, q}(a, b)=\ln b+\ln \tilde{\mathcal{T}}(p, q, t)+\ln \cos t$, we have

$$
\begin{aligned}
\frac{\partial}{\partial a} \ln \mathcal{T}_{p, q}(a, b) & =\left(\frac{\partial}{\partial t} \ln \tilde{\mathcal{T}}(p, q, t)-\frac{\sin t}{\cos t}\right) \times \frac{\partial t}{\partial a} \\
& :=-\frac{1}{\sqrt{b^{2}-a^{2}}}(J(p, q)-\tan t),
\end{aligned}
$$

where

$$
J(p, q)=\frac{j(p)-j(q)}{p-q} \quad \text { if } p \neq q \text { and } \quad J(p, p)=j^{\prime}(p)
$$

here

$$
j(x)=\frac{2 x}{\sin 2 x t} \quad \text { if }|x| \in(0,1] \quad \text { and } \quad j(0)=\frac{1}{t}
$$

is even on $[-1,1]$. Thus, to prove $\partial\left(\ln \mathcal{T}_{p, q}(a, b)\right) / \partial a>0$, it suffices to prove that for $p, q \in$ $[-1,1]$ with $0 \leq p+q \leq 1$, the inequality $J(p, q)-\tan t<0$ holds for $t \in(0, \pi / 2)$.

Utilizing (2.8) and differentiating again give

$$
\begin{aligned}
& j(x)=\frac{1}{t}+\sum_{n=1}^{\infty} \frac{\left(2^{2 n}-2\right) 2^{2 n}}{(2 n) !}\left|B_{2 n}\right| t^{2 n-1} x^{2 n}, \\
& j^{\prime \prime}(x)=\sum_{n=1}^{\infty} \frac{2 n(2 n-1)\left(2^{2 n}-2\right) 2^{2 n}}{(2 n) !}\left|B_{2 n}\right| t^{2 n-1} x^{2 n-2}>0 \quad \text { for } x \in[-1,1], \\
& j^{\prime \prime \prime}(x)=\sum_{n=2}^{\infty} \frac{2 n(2 n-1)(2 n-2)\left(2^{2 n}-2\right) 2^{2 n}}{(2 n) !}\left|B_{2 n}\right| t^{2 n-1} x^{2 n-3} \geq 0 \quad \text { for } x \in[0,1] .
\end{aligned}
$$

By Lemmas 2.1 and 2.3 we see that $J(p, q)$ is increasing in $p$ and $q$ on $[-1,1]$, and $J(p, 1-p)$ is decreasing on $[0,1 / 2)$ and increasing on $(1 / 2,1]$.

Now we distinguish two cases to prove $J(p, q)-\tan t<0$ for $p, q \in[-1,1]$ with $0 \leq p+q \leq 1$.

Case 1: $p, q \geq 0$ and $p+q \leq 1$. By the monotonicity of $J(p, q)$ in $p, q$ on $[-1,1]$ and of $J(p, 1-p)$ in $p$ on $[0,1]$, we have

$$
\begin{aligned}
J(p, q)-\tan t & \leq J(p, 1-p)-\tan t \leq J(1,0)-\tan t \\
& =\frac{2}{\sin 2 t}-\frac{1}{t}-\frac{\sin t}{\cos t}=\frac{\cos t}{\sin t}-\frac{1}{t}<0 .
\end{aligned}
$$

Case 2: $p \geq 0, q \leq 0$ or $p \leq 0, q \geq 0$ and $p+q \leq 1$. We assume that $p \geq q$. Then $p \geq 0$, $q \leq 0$ and $p \leq \min (1-q, 1)=1$. Therefore, we get

$$
J(p, q)-\tan t \leq J(1,0)-\tan t<0
$$

which proves the monotonicity of $\mathcal{T}_{p, q}(a, b)$ in $a$ on $(0, b)$. 
Remark 5.1 Utilizing the monotonicity property, we have

$$
\mathcal{T}_{1 / 2,1 / 2}(G, A)<\mathcal{T}_{1 / 2,1 / 2}(G, Q)<\mathcal{T}_{1 / 2,1 / 2}(A, Q),
$$

which indicates that

$$
G \exp \left(\frac{2 A}{P}-2\right)<G \exp \left(\frac{2 Q}{U}-2\right)<A \exp \left(\frac{2 Q}{T}-2\right)
$$

Additionally, $\mathcal{T}_{p, q}(a, b)$ has a unique property which shows the relation among twoparameter sine, cosine and tangent means.

Property 5.6 For $0<a<b$, if $0 \leq p+q \leq 1$, then $\mathcal{T}_{p, q}(a, b)=a \mathcal{S}_{p, q}(a, b) / \mathcal{C}_{p, q}(a, b)$. In particular, $\mathcal{T}_{1,0}(a, b)=\mathcal{S}_{1,0}(a, b)=S B(a, b)$.

\section{Inequalities for two-parameter trigonometric means}

As shown in the previous sections-, by using Propositions 3.1-5.2 we can establish a series of new inequalities for trigonometric functions and reprove some known ones. However, we are more interested in how to establish new inequalities for two-parameter trigonometric means from these ones derived by using Propositions 3.1-5.2, as obtaining an inequality for bivariate mans from the corresponding one for hyperbolic functions (see [22-24, 30]). In fact, Neuman also offered some successful examples (see [31]).

The inequalities involving Schwab-Borchardt mean $S B$ are mainly due to Neuman and Sándor (see [31-33]), and Witkowski [34] also has some contributions to them. More often, however, inequalities for means constructed by trigonometric functions seem to be related to the first and second Seiffert means, see [11, 33, 35-55]. In this section, we establish some new inequalities for two-parameter trigonometric means by using their monotonicity and log-convexity. Our steps are as follows.

Step 1: Obtaining an inequality $\left(\mathrm{I}_{1}\right)$ for trigonometric functions $\sin t, \cos t$ and $\tan t$ by using the monotonicity and log-convexity of two-parameter trigonometric functions and simplifying them.

Step 2: For $0<a<b$, letting $t=\arccos (a / b)$ in inequalities $\left(\mathrm{I}_{1}\right)$ obtained in Step 1 and next multiplying both sides by $b$ or $a$ and simplifying yield an inequality $\left(\mathrm{I}_{2}\right)$ for means involving trigonometric functions.

Step 3: Let $m=m(a, b)$ and $M=M(a, b)$ be two means of $a$ and $b$ with $m(a, b)<M(a, b)$ for all $a, b>0$. Making a change of variables $a \rightarrow m(a, b)$ and $b \rightarrow M(a, b)$ leads to another inequality $\left(\mathrm{I}_{3}\right)$ for means involving trigonometric functions.

Now we illustrate these steps.

\section{Example 6.1}

Step 1: For $t \in(0, \pi / 2)$, we have (3.8).

Step 2: For $0<a<b$, letting $t=\arccos (a / b)$ and next multiplying each side of (3.8) by $b$ and simplifying yield

$$
\sqrt{a S B(a, b)}<\frac{2 a+b}{3}<b e^{a / S B(a, b)-1}<\frac{a+b}{2}<S B(a, b)<b^{2 / 3}\left(\frac{a+b}{2}\right)^{1 / 3} .
$$


Step 3: With $(a, b) \rightarrow(G, A)$ yields

$$
\sqrt{G P}<\frac{2 G+A}{3}<A e^{G / P-1}<\frac{A+G}{2}<P<A^{2 / 3}\left(\frac{A+G}{2}\right)^{1 / 3} .
$$

With $(a, b) \rightarrow(G, Q)$ yields

$$
\sqrt{G U}<\frac{2 G+Q}{3}<Q e^{G / U-1}<\frac{G+Q}{2}<U<Q^{2 / 3}\left(\frac{G+Q}{2}\right)^{1 / 3} .
$$

With $(a, b) \rightarrow(A, Q)$ yields

$$
\sqrt{A T}<\frac{2 A+Q}{3}<Q e^{A / T-1}<\frac{A+Q}{2}<T<Q^{2 / 3}\left(\frac{A+Q}{2}\right)^{1 / 3}
$$

\section{Example 6.2}

Step 1: For $t \in(0, \pi / 2)$, from (3.9) it is derived that

$$
\begin{aligned}
\cos ^{1 / 3} t & <\left(\frac{2}{3} \cos t+\frac{1}{3}\right)^{1 / 2}<\frac{\sin t}{t}<\left(\frac{2}{3} \cos \frac{t}{2}+\frac{1}{3}\right)^{2} \\
& <e^{t(1+\cos t) / \sin t-2}<\left(\frac{1}{2}+\frac{1}{2} \cos \frac{t}{2}\right)^{2} .
\end{aligned}
$$

Step 2: For $0<a<b$, letting $t=\arccos (a / b)$ and next multiplying each side of (6.1) by $b$ and simplifying yield

$$
\begin{aligned}
b^{2 / 3} a^{1 / 3} & <b^{1 / 2}\left(\frac{2}{3} a+\frac{1}{3} b\right)^{1 / 2}<S B(a, b) \\
& <\left(\frac{2}{3} \sqrt{\frac{a+b}{2}}+\frac{\sqrt{b}}{3}\right)^{2}<b e^{(a+b) / S B(a, b)-2} .
\end{aligned}
$$

Step 3: With $(a, b) \rightarrow(G, A)$ yields

$$
A^{2 / 3} G^{1 / 3}<A^{1 / 2}\left(\frac{2}{3} G+\frac{1}{3} A\right)^{1 / 2}<P<\left(\frac{2}{3} \sqrt{\frac{A+G}{2}}+\frac{1}{3} \sqrt{A}\right)^{2}<A e^{(A+G) / P-2} .
$$

With $(a, b) \rightarrow(G, Q)$ yields

$$
Q^{2 / 3} G^{1 / 3}<Q^{1 / 2}\left(\frac{2}{3} G+\frac{1}{3} Q\right)^{1 / 2}<U<\left(\frac{2}{3} \sqrt{\frac{G+Q}{2}}+\frac{1}{3} \sqrt{Q}\right)^{2}<Q e^{(G+Q) / U-2}
$$

With $(a, b) \rightarrow(A, Q)$ yields

$$
Q^{2 / 3} A^{1 / 3}<Q^{1 / 2}\left(\frac{2}{3} A+\frac{1}{3} Q\right)^{1 / 2}<T<\left(\frac{2}{3} \sqrt{\frac{A+Q}{2}}+\frac{1}{3} \sqrt{Q}\right)^{2}<Q e^{(A+Q) / T-2}
$$

\section{Example 6.3}

Step 1: For $t \in(0, \pi / 2)$, we have (4.3). 
Step 2: For $0<a<b$, letting $t=\arccos (a / b)$ and next multiplying each side of (4.3) by $b$ and simplifying yield

$$
\frac{a+b}{2}>b e^{(a-b) / S B(a, b)}>\left(2 \sqrt{\frac{a+b}{2}}-\sqrt{b}\right)^{2}>a .
$$

Step 3: With $(a, b) \rightarrow(G, A)$ yields

$$
\frac{A+G}{2}>A e^{(G-A) / P}>\left(2 \sqrt{\frac{A+G}{2}}-\sqrt{A}\right)^{2}>G
$$

With $(a, b) \rightarrow(G, Q),(A, Q)$ can yield corresponding inequalities.

Remark 6.1 From inequalities (6.3) it is derived that

$$
L(a, b)<S B(a, b)<2 L\left(\frac{a+b}{2}, b\right)
$$

hold for $0<a<b$, where $L(x, y)$ is the logarithmic mean of positive numbers $x$ and $y$. The first inequality of (6.5) follows from the relation between the second and fourth terms, that is, $b \exp ((a-b) / S B(a, b))>a$, while the second one is obtained by the first one in (6.3).

\section{Example 6.4}

Step 1: For $t \in(0, \pi / 2)$, we have (5.4).

Step 2: For $0<a<b$, letting $t=\arccos (a / b)$ and next multiplying each side of (5.4) by $a$ and simplifying give

$$
a\left(\frac{S B(a, b)}{\frac{a+b}{2}}\right)^{2}<a e^{2 b / S B(a, b)-2}<a \frac{\left(\frac{2}{3} \sqrt{\frac{a+b}{2}}+\frac{1}{3} \sqrt{b}\right)^{4}}{\left(\frac{2}{3} a+\frac{1}{3} b\right)^{2}}<S B(a, b)
$$

Step 3: With $(a, b) \rightarrow(G, A)$ yields

$$
G\left(\frac{2 P}{A+G}\right)^{2}<G e^{2 A / P-2}<G \frac{\left(\frac{2}{3} \sqrt{\frac{A+G}{2}}+\frac{1}{3} \sqrt{A}\right)^{4}}{\left(\frac{2}{3} G+\frac{1}{3} A\right)^{2}}<P
$$

With $(a, b) \rightarrow(G, Q),(A, Q)$, we can derive corresponding inequalities.

Remark 6.2 Applying our method in establishing inequalities for means to certain known ones involving trigonometric functions, we can obtain corresponding inequalities which are possibly related to means. For example, the Wilker inequality states that for $t \in(0, \pi / 2)$,

$$
\left(\frac{\sin t}{t}\right)^{2}+\frac{\tan t}{t}>2
$$

If for $0<a<b$, put $t=\arccos (a / b)$, then we have

$$
\left(\frac{S B(a, b)}{b}\right)^{2}+\frac{S B(a, b)}{a}>2 .
$$


In a similar way, the following inequalities

$$
\left(\frac{1+\cos t}{2}\right)^{2 / 3}<\frac{\sin t}{t}<\frac{2+\cos t}{3}, \quad t \in(0, \pi / 2)
$$

can be changed into

$$
b^{1 / 3}\left(\frac{a+b}{2}\right)^{2 / 3}<S B(a, b)<\frac{2 b+a}{3}
$$

by letting $t=\arccos (a / b)$ for $0<a<b$, where the left inequality in (6.8) is due to Neuman and Sándor [56, (2.5)] (also see [57-59]) and the right one is known as Cusa's inequality.

Remark 6.3 The third inequality in (6.1) is clearly superior to Cusa's inequality (the right one of (6.8)) because

$$
\left(\frac{2}{3} \cos \frac{t}{2}+\frac{1}{3}\right)^{2}<\frac{2}{3} \cos ^{2} \frac{t}{2}+\frac{1}{3}=\frac{2+\cos t}{3}
$$

While the second one in (6.1) is weaker than the first one in (6.8) since

$$
\left(\frac{2}{3} \cos t+\frac{1}{3}\right)^{3}-\left(\frac{1+\cos t}{2}\right)^{4}=-\frac{1}{432}\left(34 \cos t+27 \cos ^{2} t+11\right)(\cos t-1)^{2}<0
$$

\section{Families of two-parameter hyperbolic means}

After three families of two-parameter trigonometric means have been successfully constructed, we are encouraged to establish further two-parameter means of a hyperbolic version. They are included in the following theorems.

Theorem 7.1 Let $p, q \in \mathbb{R}$, and let $\operatorname{Sh}(p, q, t)$ be defined by (3.1). Then, for all $a, b>0$, $S h_{p, q}(b, a)$ defined by

$$
\begin{aligned}
& \operatorname{Sh}_{p, q}(b, a)=\min (a, b) \times \operatorname{Sh}\left(p, q, \operatorname{arccosh}\left(\frac{\max (a, b)}{\min (a, b)}\right)\right) \quad \text { if } a \neq b \text { and } \\
& S h_{p, q}(a, a)=a
\end{aligned}
$$

is $a$ mean of $a$ and $b$ if and only if

$$
\begin{aligned}
& p+q \leq 3 \quad \text { and } \quad L(p, q) \leq \frac{1}{\ln 2}, \quad \text { if } p, q>0 \text {, } \\
& 0 \leq p+q \leq 3 \text {, otherwise. }
\end{aligned}
$$

Theorem 7.2 Let $p, q \in \mathbb{R}$, and let $C h(p, q, t)$ be defined by (4.1). Then, for all $a, b>0$, $C h_{p, q}(b, a)$ defined by

$$
\begin{aligned}
& C h_{p, q}(b, a)=\min (a, b) \times C h\left(p, q, \operatorname{arccosh}\left(\frac{\max (a, b)}{\min (a, b)}\right)\right) \quad \text { if } a \neq b \text { and } \\
& C h_{p, q}(a, a)=a
\end{aligned}
$$

is a mean of $a$ and $b$ if and only if $0 \leq p+q \leq 1$. 
To prove the above theorems, it suffices to use comparison theorems given in $[15,16]$ by Páles because both $S h_{p, q}(b, a)$ and $C h_{p, q}(b, a)$ are means if and only if

$$
\begin{aligned}
& \operatorname{Sh}(0,0, t)=1 \leq \operatorname{Sh}(p, q, t) \leq \cosh t=\operatorname{Sh}(2,1, t), \\
& \operatorname{Ch}(0,0, t)=1 \leq \operatorname{Ch}(p, q, t) \leq \cosh t=\operatorname{Ch}(1,0, t),
\end{aligned}
$$

respectively. Here we omit further details.

The monotonicities and log-convexities of $S h_{p, q}(b, a)$ and of $C h_{p, q}(b, a)$ in parameters $p$ and $q$ are clearly the same as those of $\operatorname{Sh}(p, q, t)$ and of $\operatorname{Ch}(p, q, t)$, which are in turn equivalent to those of Stolarsky means defined by (1.1) and of Gini means defined by (1.2), respectively. These properties can be found in [13, 14, 17-19, 21].

The above theorems indicate that for $0<a<b$, all the following

$$
\begin{aligned}
& S h_{1,0}(b, a)=a \frac{\sinh t}{t}=\frac{\sqrt{b^{2}-a^{2}}}{\operatorname{arccosh}(b / a)}=S B(b, a), \\
& S h_{1,1}(b, a)=a \exp (t \operatorname{coth} t-1)=a \exp \left(\frac{b}{S B(b, a)}-1\right), \\
& C h_{1 / 2,1 / 2}(b, a)=a \exp \left(t \tanh \frac{t}{2}\right)=a \exp \left(t \frac{\cosh t-1}{\sinh t}\right)=a \exp \left(\frac{b-a}{S B(b, a)}\right)
\end{aligned}
$$

are means of $a$ and $b$, where $S B(b, a)$ is the Schwab-Borchardt mean defined by (1.3).

It is easy to verify that

$$
\begin{aligned}
& S h_{1,0}(A, G)=S B(A, G)=\frac{b-a}{\ln b-\ln a}=L(b, a), \\
& S h_{1,1}(A, G)=G \exp \left(\frac{A}{L}-1\right)=I(b, a), \\
& C h_{1 / 2,1 / 2}(A, G)=G \exp \left(\frac{A-G}{L}\right)=Z_{1 / 2}(b, a),
\end{aligned}
$$

where $L(b, a)$ and $I(b, a)$ are logarithmic and identric means, respectively, while $Z_{p}(b, a)=$ $Z^{1 / p}\left(a^{p}, b^{p}\right)$ is the $p$-order power-exponential mean. Also, all the following

$$
\begin{aligned}
& S h_{1,0}(Q, G)=S B(Q, G)=\frac{a-b}{\sqrt{2} \operatorname{arcsinh} \frac{a-b}{\sqrt{2 a b}}}:=V(a, b), \\
& S h_{1,1}(Q, G)=G \exp \left(\frac{Q}{V}-1\right), \\
& C h_{1 / 2,1 / 2}(Q, G)=G \exp \left(\frac{Q-G}{V}\right)
\end{aligned}
$$

are means lying in $G$ and $Q$. Likewise, all the following

$$
\begin{aligned}
& S h_{1,0}(Q, A)=S B(Q, A)=\frac{a-b}{2 \operatorname{arcsinh} \frac{a-b}{a+b}}=N S(a, b), \\
& S h_{1,1}(Q, A)=A \exp \left(\frac{Q}{N S}-1\right),
\end{aligned}
$$




$$
C h_{1 / 2,1 / 2}(Q, A)=A \exp \left(\frac{Q-A}{N S}\right)
$$

are also means between $A$ and $Q$, where $N S(a, b)$ is the Neuman-Sándor mean defined by (1.4).

It should be noted that the new mean $V(a, b)$ is similar to $N S(a, b)$.

Similar to (5.1), for $p, q \in \mathbb{R}$, we can define the two-parameter hyperbolic tangent function as follows:

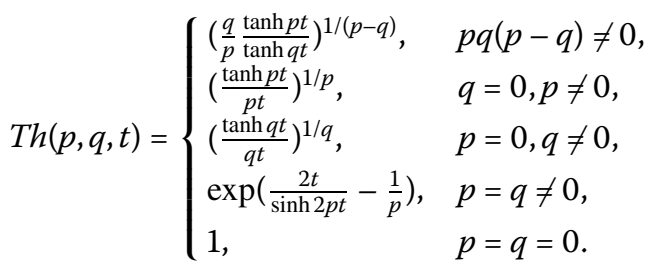

By some verifications, however, $T h(p, q, t)$ does not have good properties like monotonicity in parameters $p$ and $q$, and therefore, we fail to define a family of two-parameter hyperbolic tangent means. However, for certain $p, q$ and $0<a<b$, it is showed that $b T h(p, q, \operatorname{arccosh}(b / a))$ is a mean of $a$ and $b$, for example,

$$
b \times \operatorname{Th}(1,0, \operatorname{arccosh}(b / a))=S B(b, a)
$$

is clearly a mean of $a$ and $b$. It is also proved that

$$
b \times \operatorname{Th}\left(\frac{1}{2}, \frac{1}{2}, \operatorname{arccosh}(b / a)\right)
$$

is also a mean of $a$ and $b$. For this reason, we pose an open problem as the end of this paper.

Problem 7.1 Let $0<a<b$, and let $T h(p, q, t)$ be defined by (7.3). Finding $p, q$ such that $b \times \operatorname{Th}(p, q, \operatorname{arccosh}(b / a))$ is a mean of $a$ and $b$.

\section{Competing interests}

The author declares that they have no competing interests.

\section{Acknowledgements}

The author would like to thank Ms. Jiang Yiping for her help. The author also wishes to thank the reviewer(s) who gave some important and valuable advice.

Received: 16 April 2013 Accepted: 10 October 2013 Published: 19 Nov 2013

\section{References}

1. Stolarsky, KB: Generalizations of the logarithmic mean. Math. Mag. 48, 87-92 (1975)

2. Gini, C: Di una formula comprensiva delle medie. Metron 13, 3-22 (1938)

3. Brenner, JL, Carlson, BC: Homogeneous mean values: weights and asymptotes. J. Math. Anal. Appl. 123, 265-280 (1987)

4. Carlson, BC: Algorithms involving arithmetic and geometric means. Am. Math. Mon. 78, 496-505 (1971)

5. Neuman, E, Sándor, J: On the Schwab-Borchardt mean. Math. Pannon. 14(2), 253-266 (2003)

6. Toader, G: Some mean values related to the arithmetic-geometric mean. J. Math. Anal. Appl. 218(2), 358-368 (1998)

7. Sándor, J, Toader, G: On some exponential means. Seminar on Mathematical Analysis (Cluj-Napoca, 1989-1990), Preprint, 90, "Babes-Bolyai” Univ., Cluj, 35-40 (1990)

8. Sándor, J, Toader, G: On some exponential means. Part II. Int. J. Math. Math. Sci. 2006, Article ID 51937 (2006) 
9. Seiffert, HJ: Werte zwischen dem geometrischen und dem arithmetischen Mittel zweier Zahlen. Elem. Math. 42, 105-107 (1987)

10. Seiffert, HJ: Aufgabe 16. Die Wurzel 29, 221-222 (1995)

11. Sándor, J: Trigonometric and hyperbolic inequalities. Available online at arXiv:1105.0859 (2011)

12. Sándor, J: Two sharp inequalities for trigonometric and hyperbolic functions. Math. Inequal. Appl. 15(2), 409-413 (2012)

13. Leach, EB, Sholander, MC: Extended mean values. Am. Math. Mon. 85, 84-90 (1978)

14. Leach, EB, Sholander, MC: Extended mean values II. J. Math. Anal. Appl. 92, 207-223 (1983)

15. Páles, Z: Inequalities for sums of powers. J. Math. Anal. Appl. 131, 265-270 (1988)

16. Páles, Z: Inequalities for differences of powers. J. Math. Anal. Appl. 131, 271-281 (1988)

17. Qi, F: Logarithmic convexities of the extended mean values. Proc. Am. Math. Soc. 130(6), 1787-1796 (2002)

18. Yang, Z-H: On the homogeneous functions with two parameters and its monotonicity. J. Inequal. Pure Appl. Math. 6(4), Article ID 101 (2005). Available online at http://www.emis.de/journals/JIPAM/images/155_05_JIPAM/155_05.pdf

19. Yang, Z-H: On the log-convexity of two-parameter homogeneous functions. Math. Inequal. Appl. 10(3), 499-516 (2007)

20. Yang, Z-H: On the monotonicity and log-convexity of a four-parameter homogeneous mean. J. Inequal. Appl. 2008, Article ID 149286 (2008). Available online at http://www.journalofinequalitiesandapplications.com/content/2008/1/149286

21. Yang, Z-H: The log-convexity of another class of one-parameter means and its applications. Bull. Korean Math. Soc. 49(1), 33-47 (2012). Available online at http://www.mathnet.or.kr/mathnet/thesis_file/BKMS-49-1-33-47.pdf

22. Yang, Z-H: New sharp bounds for logarithmic mean and identric mean. J. Inequal. Appl. (2013). doi:10.1186/1029-242X-2013-116

23. Zhu, L: Generalized Lazarevićs inequality and its applications - Part II. J. Inequal. Appl. 2009, Article ID 379142 (2009)

24. Zhu, L: New inequalities for hyperbolic functions and their applications. J. Inequal. Appl. (2012). doi:10.1186/1029-242X-2012-303

25. Bullen, PS: Handbook of Means and Their Inequalities. Kluwer Academic, Dordrecht (2003)

26. Merkle, M: Conditions for convexity of a derivative and some applications to the Gamma function. Aequ. Math. 55, 273-280 (1998)

27. Mitrinović, DS: Analytic Inequalities. Springer, Berlin (1970)

28. Mitrinović, DS: Elementary Inequalities. Noordhoff, Groningen (1964)

29. Group of compilation: Handbook of Mathematics. Peoples' Education Press, Beijing (1979) (Chinese)

30. Yang, Z-H: New sharp bounds for identric mean in terms of logarithmic mean and arithmetic mean. J. Math. Inequal. 6(4), 533-543 (2012). doi:10.7153/jmi-06-51

31. Neuman, E: Inequalities for the Schwab-Borchardt mean and their applications. J. Math. Inequal. 5(4), 601-609 (2011)

32. Neuman, E, Sándor, J: On certain means of two arguments and their extensions. Int. J. Math. Math. Sci. 16, 981-993 (2003)

33. Neuman, E, Sándor, J: On the Schwab-Borchardt mean. Math. Pannon. 17(1), 49-59 (2006)

34. Witkowski, A: Interpolations of Schwab-Borchardt mean. Math. Inequal. Appl. 16(1), 193-206 (2012)

35. Jagers, AA: Solution of problem 887. Nieuw Arch. Wiskd. 12(2), 30-31 (1994)

36. Sándor, J: On certain inequalities for means III. Arch. Math. 76, 34-40 (2001)

37. Hästö, PA: A monotonicity property of ratios of symmetric homogeneous means. J. Inequal. Pure Appl. Math. 3(5), Article ID 71 (2002)

38. Sándor, J, Neuman, E: On certain means of two arguments and their extensions. Int. J. Math. Math. Sci. 2003(16), 981-993 (2003)

39. Chu, Y-M, Qiu, Y-F, Wang, M-K: Sharp power mean bounds for the combination of Seiffert and geometric means. Abstr. Appl. Anal. 2010, Article ID 108920 (2010)

40. He, D, Shen, Z-J: Advances in research on Seiffert mean. Commun. Inequal. Res. 17(4), Article ID 26 (2010). Available online at http://old.irgoc.org/Article/UploadFiles/201010/20101026104515652.pdf

41. Wang, S-S, Chu, Y-M: The best bounds of the combination of arithmetic and harmonic means for the Seiffert's mean. Int. J. Math. Anal. 4(21-24), 1079-1084 (2010)

42. Wang, M-K, Qiu, Y-F, Chu, Y-M: Sharp bounds for Seiffert means in terms of Lehmer means. J. Math. Inequal. 4(4), 581-586 (2010)

43. Chu, Y-M, Qiu, Y-F, Wang, M-K, Wang, G-D: The optimal convex combination bounds of arithmetic and harmonic means for the Seiffert's mean. J. Inequal. Appl. 2010, Article ID 436457 (2010)

44. Liu, H, Meng, X-J: The optimal convex combination bounds for Seiffert's mean. J. Inequal. Appl. 2011, Article ID $686834(2011)$

45. Chu, Y-M, Wang, M-K, Gong, W-M: Two sharp double inequalities for Seiffert mean. J. Inequal. Appl. 2011, 44 (2011) doi:10.1186/1029-242X-2011-44

46. Chu, Y-M, Hou, S-W: Sharp bounds for Seiffert mean in terms of contraharmonic mean. Abstr. Appl. Anal. 2012, Article ID $425175(2012)$

47. Chu, Y-M, Long, B-Y, Gong, W-M, Song, Y-Q: Sharp bounds for Seiffert and Neuman-Sándor means in terms of generalized logarithmic means. J. Inequal. Appl. 2013, 10 (2013). Available online at http://www.journalofinequalitiesandapplications.com/content/2013/1/10

48. Jiang, W-D, Qi, F: Some sharp inequalities involving Seiffert and other means and their concise proofs. Math. Inequal. Appl. 15(4), 1007-1017 (2012)

49. Hästö, PA: Optimal inequalities between Seiffert's mean and power mean. Math. Inequal. Appl. 7(1), 47-53 (2004)

50. Costin, I, Toader, G: A nice separation of some Seiffert type means by power means. Int. J. Math. Math. Sci. 2012, Article ID 430692 (2012)

51. Yang, Z-H: Sharp bounds for the second Seiffert mean in terms of power means. Available online at arXiv:1206.5494v1 (2012)

52. Yang, Z-H: The monotonicity results and sharp inequalities for some power-type means of two arguments. Available online at arXiv:1210.6478 (2012) 
53. Yang, Z-H: Sharp bounds for Seiffert mean in terms of weighted power means of arithmetic mean and geometric mean. Math. Inequal. Appl. (2013, in print)

54. Chu, Y-M, Wang, M-K, Qiu, Y-F: An optimal double inequality between power-type Heron and Seiffert means. J. Inequal. Appl. 2010, Article ID 146945 (2010)

55. Costin, I, Toader, G: Optimal evaluations of some Seiffert-type means by power means. Appl. Math. Comput. 219, 4745-4754 (2013). doi:10.1016/j.amc.2012.10.091

56. Neuman, E, Sándor, J: On some inequalities involving trigonometric and hyperbolic functions with emphasis on the Cusa-Huygens, Wilker and Huygens inequalities. Math. Inequal. Appl. 13(4), 715-723 (2010)

57. LV, Y-P, Wang, G-D, Chu, Y-M: A note on Jordan type inequalities for hyperbolic functions. Appl. Math. Lett. 25, 505-508 (2012)

58. Yang, Z-H: Refinements of Mitrinovic-Cusa inequality. Available online at arXiv:1206.4911 (2012)

59. Yang, Z-H: Refinements of a two-sided inequality for trigonometric functions. J. Math. Inequal. 7(4), $601-615$ (2013). doi:10.7153/jmi-07-57

10.1186/1029-242X-2013-541

Cite this article as: Yang: Three families of two-parameter means constructed by trigonometric functions. Journal of Inequalities and Applications 2013, 2013:541

\section{Submit your manuscript to a SpringerOpen ${ }^{\circ}$ journal and benefit from:}

- Convenient online submission

- Rigorous peer review

- Immediate publication on acceptance

- Open access: articles freely available online

- High visibility within the field

- Retaining the copyright to your article 